\title{
Triangular titanium implants for sacroiliac joint fusion
}

\author{
Nikolai G. Rainov ${ }^{1} \cdot$ Reinhard Schneiderhan ${ }^{1} \cdot$ Volkmar Heidecke $^{2}$
}

Received: 16 September 2018 / Revised: 9 December 2018 / Accepted: 12 December 2018 / Published online: 18 December 2018 (c) The Author(s) 2018

\begin{abstract}
Background Sacroiliac joint (SIJ) dysfunction is an underdiagnosed condition. Several published cohorts have reported favorable midterm outcomes after SIJ fusion using titanium implants placed across the SIJ. Herein, we report 12-month follow-up from SIJ fusion in a standard clinic setting.

Methods A carefully selected group of 160 consecutive patients with painful SIJ dysfunction were diagnosed at a single center and underwent unilateral or staged bilateral SIJ fusion using triangular titanium implants. Patients were routinely seen in clinic for follow-up every 3 months where they completed visual analog scale (0-10 range) pain ratings and Oswestry Disability Index (ODI). Follow-up CT scan was performed at 1 year.

Results Mean patient age was 58 years, and $68 \%$ were women. $30 \%$ underwent staged bilateral SIJ fusion. By month 12, SIJ pain decreased from 8.0 to $2.5(p<0.0001)$ and disability (ODI) decreased from 45.3 to $16.4(p<0.0001)$. The proportion with clinically significant improvements in SIJ pain and ODI was high $(>95 \%)$. Perioperative adverse events were mild and decreased with increasing surgical experience with the procedure. Subgroup analysis showed slightly smaller improvements in those undergoing bilateral surgery and those with a spinal cord stimulator in place. CT scan at 1 year showed reabsorption along one or more implants in $16 \%$ of cases, but there were no breakages or implant removals.

Conclusions In standard clinical practice, SIJ fusion with triangular titanium implants produces significant improvement in pain and disability related to SIJ dysfunction.
\end{abstract}

Graphical abstract These slides can be retrieved under Electronic Supplementary Material.

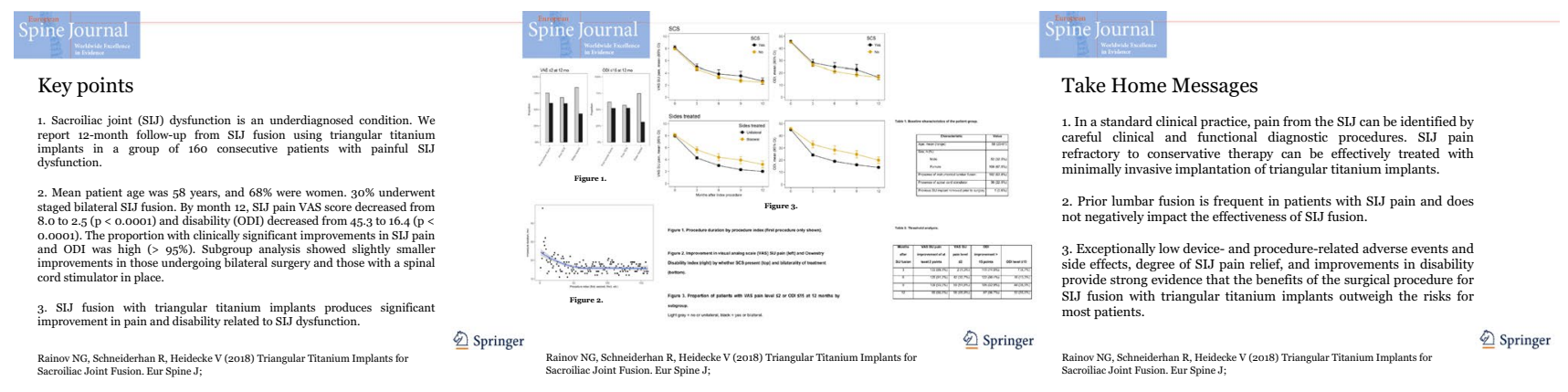

Keywords Minimally invasive surgery $\cdot$ Sacroiliac joint dysfunction $\cdot$ Sacroiliac joint disruptions $\cdot$ Degenerative sacroiliitis $\cdot$ Sacroiliac joint arthrodesis $\cdot$ Spine surgery

Electronic supplementary material The online version of this article (https://doi.org/10.1007/s00586-018-5860-1) contains supplementary material, which is available to authorized users.

Nikolai G. Rainov

rainov@orthopaede.com

Extended author information available on the last page of the article

\section{Background}

Chronic low back pain originating in the sacroiliac joint (SIJ) is common, accounting for $15-30 \%$ of all cases of low back pain [1-5]. SIJ pain is debilitating and associated with low quality of life (QOL) [6, 7]. 
Non-surgical treatments for SIJ pain are commonly provided, including intra-articular steroid injections and physical therapy. However, little evidence supports the use of steroid injections as a durable treatment and they often fail to provide long-term pain relief, although they may be useful in the diagnostic workup for differentiating SIJ pain from confounding factors such as pain generated in the lumbar spine $[8,9]$. Open fusion of the SIJ was first reported in the early 1900s [10, 11], and scattered reports of patient success have been published since then [12]. Devices for minimally invasive SIJ fusion are now available, with the most studied device being triangular titanium implants. Evidence for these devices includes two randomized trials [13, 14]: a prospective multicenter trial [15] and several comparative cohorts $[16,17]$.

Herein, we report our experience with SIJ fusion using the same device in a standard clinical setting in Germany. We focus on the occurrence of bilateral symptoms and the relationship to prior lumbar fusion and implanted devices for treatment of chronic back and leg pain, which were common in our cohort.

\section{Methods}

This is a retrospective case series of patients with SIJ pain who underwent unilateral or bilateral SIJ fusion.

\section{Patients}

Patients with chronic low back pain and leg pain were evaluated in our multispecialty clinic using a standardized algorithm. All patients had a prior conservative treatment for their pain consisting of physiotherapy and/or manual therapy, oral nonsteroidal anti-inflammatory drugs and/or opioids, and SIJ and/or lumbar facet joint injections with local anesthetic and/or steroid drugs, which in all cases failed to produce long-term relief. When SIJ pain was suspected on the basis of historical findings (off-center pain below L5, possible radiation into the legs, groin pain, and difficulty sitting on the affected side or on both sides), we performed physical examination maneuvers that stress the SIJ, including compression test, Gaenslen test, thigh thrust, Patrick's test, and distraction test. Three or more examination findings have reasonable specificity for SIJ pain [9]. These are the same tests used in prospective trials of SIJ fusion. Patients with three or more positive tests underwent one or more confirmatory SIJ blocks.

Confirmatory diagnostic block was also standardized as follows: Under C-arm control, the caudal pole of the SIJ was cannulated, and local anesthetic (5 cc of ropivacaine $7.5 \%$ ) mixed with corticosteroid (triamcinolone $20 \mathrm{mg}$ ) was injected into the caudal recess of the SIJ. Patients with a marked acute reduction in pain ( $>50 \%)$ were considered to have confirmed SIJ pain. A CT scan of the lumbar spine and pelvis was performed in all cases as a part of the diagnostic workup in order to rule out other prominent sources of pain. All patients who satisfied our diagnostic criteria went on to receive SIJ fusion, except for a few cases where patients refused any surgical treatment.

Patients with bilateral pain suspected to be from the SIJ were confirmed using bilateral SIJ blocks. Patients who developed contralateral SIJ pain after surgery for singlesided pain were confirmed through similar diagnostic evaluation, including a confirmatory contralateral diagnostic SIJ blocks.

\section{Procedure}

Preoperatively, all patients underwent a CT scan of the pelvis. SIJ fusion was performed under fluoroscopic guidance with placement of 2-3 triangular titanium implants (SIBONE, Inc., Santa Clara, CA, USA) across the SIJ using a lateral transiliac approach involving drilling, broaching, and malleting implants into place. Patients were typically discharged 2 days after the procedure. Postoperatively, patients were asked to decrease weight-bearing on the surgery side as tolerated. Postoperative rehabilitation was routinely recommended starting 4 weeks after surgery.

\section{Follow-up}

Patients undergoing surgery were routinely seen in clinic postoperatively every 3 months. At each visit, they completed visual analog score (VAS) for SIJ pain and Oswestry Disability Index [18]. Some patients completed some of the VAS and ODI questionnaires at home and sent them to us by mail. Patients were evaluated for adverse events. At 1 year, patients underwent a high-resolution CT scan of the pelvis to look for radiographic evidence of malposition or implant loosening (radiolucency). We report herein the 12-month follow-up to date.

\section{Statistical analysis}

Data were entered into Microsoft Excel and analyzed using $\mathrm{R}$ [19]. Improvement in continuous measures (VAS scores and ODI) was analyzed using Student's $t$ test. Proportions and confidence intervals were calculated using exact binomial tests. Subgroup analysis by clinical characteristics was performed with differences in responses judged using repeated measures analysis of variance. The proportions of patients with VAS pain ratings improving by 2 or more points and the proportion of patients with ODI improvement of 15 or more points were compared using Fisher tests. 


\section{Results}

After screening of all low back pain patients in our clinic from 2015 to 2017, 160 consecutive patients diagnosed with typical SIJ pain resistant to conservative treatments underwent SIJ fusion. Mean age was 58 years, and $68 \%$ were women. Prior instrumented lumbar fusion was common in our cohort (64\%), and $23 \%$ also had a spinal cord stimulator (SCS) in place (Table 1). Patients undergoing bilateral surgery were more likely younger $(p=0.0004)$ but had no other differences in terms of sex, the presence of prior lumbar fusion, or the presence of SCS.

\section{Procedure characteristics}

Forty-eight patients underwent contralateral SIJ fusion, with $36(75 \%)$ of these having bilateral SIJ pain at baseline. All surgeries were performed in a highly standardized manner by one senior neurosurgeon with $>25$ years of clinical experience. Procedure time (first incision to last suture) averaged

Table 1 Baseline characteristics of the patient group

\begin{tabular}{lc}
\hline Characteristic & \multicolumn{1}{l}{ Value } \\
\hline Age, mean (range) & $58(20-91)$ \\
Sex, $n(\%)$ & \\
Male & $52(32.5 \%)$ \\
Female & $108(67.5 \%)$ \\
Presence of instrumented lumbar fusion & $102(63.8 \%)$ \\
Presence of spinal cord stimulator & $36(22.5 \%)$ \\
Previous SIJ implant removed prior to surgery & $1(0.6 \%)$ \\
\hline
\end{tabular}

$17 \mathrm{~min}$ (first procedure, range 11-48 $\mathrm{min}$ ) and $15 \mathrm{~min}$ (second procedure, range 12-24 $\mathrm{min}$ ). There were minor variations in mean procedure time by baseline predictors, with variations of $<2 \mathrm{~min}$ by each factor. Procedure time generally decreased with gaining experience, and procedures after the first 25 cases generally lasted less than 20 min (Fig. 1). During the first procedure, three implants were used in 37 cases $(23 \%)$ and two implants in 123 cases $(77 \%)$.

There were no intraoperative complications. Postoperatively, we observed a small number of patients (typically older patients) with distended soft tissues who developed painful hematomas in the surgical path of approach. This complication occurred within the first 25 cases of the cohort and was virtually eradicated after routinely applying compression to the surgical site using an elastic bandage for 12-24 h. There were no postoperative wound infections, implant revisions due to implant malposition, and no displacements or pullouts of SIJ implants.

Follow-up was obtained in 151 patients at 3 months, 135 patients at 6 months, 114 at 9 months, and 90 at 12 months. Mean $( \pm$ SD) VAS of SIJ pain improved from $8.0( \pm 0.8)$ at baseline to $3.5( \pm 1.6)$ at 6 months and $2.5( \pm 1.4)$ at 12 months ( $p<0.0001$ for change from baseline). Mean (SD) ODI improved from $45.3( \pm 2.3)$ at baseline to $22.1( \pm 8.8)$ at 6 months and $16.4( \pm 6.6)$ at 12 months ( $p<0.0001$ vs. baseline). In the group of patients followed up for 12 months, 97\% had a VAS improvement from baseline of at least 2 points, $97 \%$ had ODI improvement of at least 15 points, $66 \%$ had a VAS level of 2 or less, and $56 \%$ had an ODI level of 15 or less (Table 2 ).

More than $50 \%$ of patients had a 12-month VAS level $\leq 2$ or ODI $\leq 15$ independent of whether there was prior lumbar fusion or SCS in place (Fig. 2). For patients with bilateral
Fig. 1 Procedure duration by procedure index (first procedure only shown)

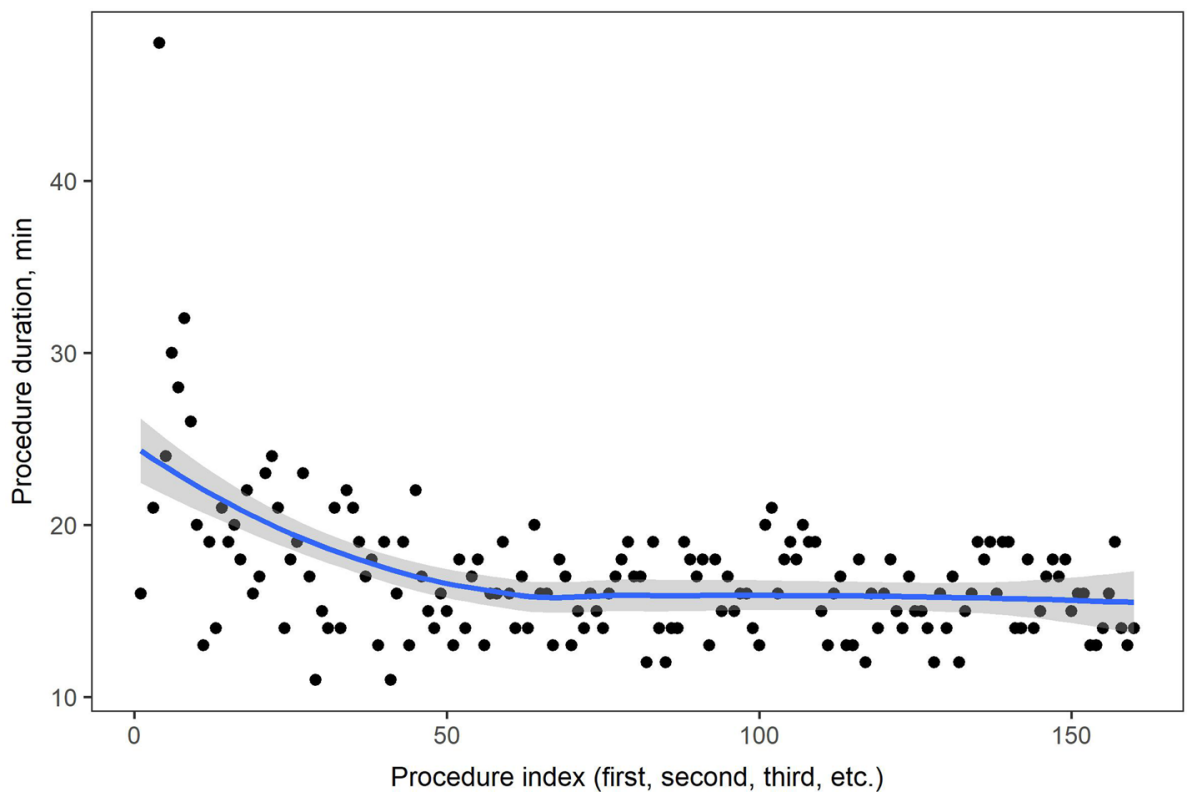


Table 2 Threshold analysis

\begin{tabular}{lcccc}
\hline $\begin{array}{l}\text { Months after } \\
\text { SIJ fusion }\end{array}$ & $\begin{array}{l}\text { VAS SIJ pain improve- } \\
\text { ment of at least 2 points }\end{array}$ & VAS SIJ pain level $\leq 2$ & $\begin{array}{l}\text { ODI improve- } \\
\text { ment }>15 \text { points }\end{array}$ & ODI level $\leq 15$ \\
\hline 3 & $133(88.1 \%)$ & $2(1.3 \%)$ & $111(74.5 \%)$ & $7(4.7 \%)$ \\
6 & $125(91.2 \%)$ & $42(30.7 \%)$ & $122(90.4 \%)$ & $18(13.3 \%)$ \\
9 & $106(93.0 \%)$ & $59(51.8 \%)$ & $105(92.9 \%)$ & $44(38.9 \%)$ \\
12 & $85(96.6 \%)$ & $58(65.9 \%)$ & $87(96.7 \%)$ & $50(55.6 \%)$ \\
\hline
\end{tabular}
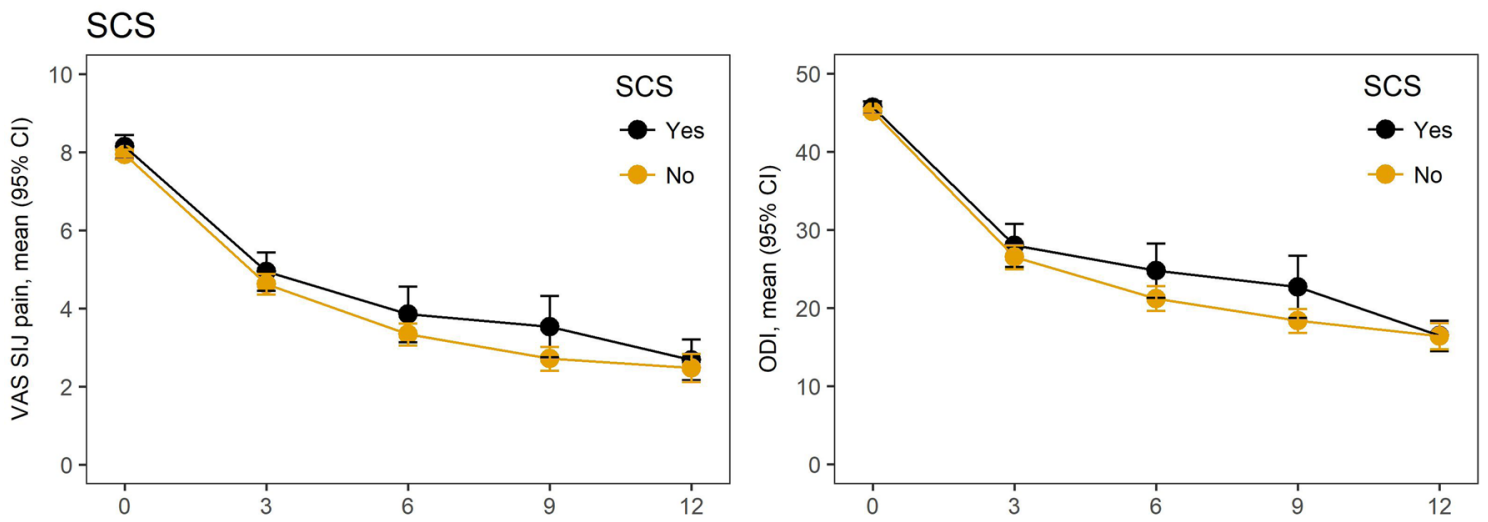

Sides treated
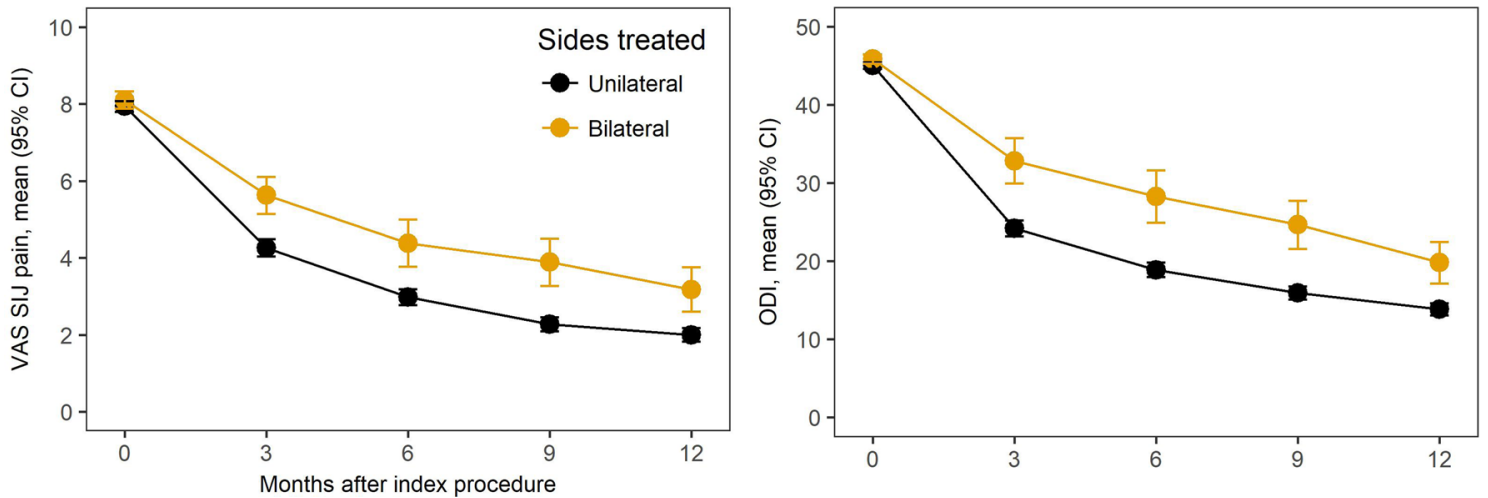

Fig. 2 Improvement in visual analog scale (VAS) SIJ pain (left) and Oswestry Disability Index (right) by whether SCS present (top) and bilaterality of treatment (bottom)

treatment, the proportion with 12-month VAS $\leq 2$ was $44 \%$ and the proportion with 12 -month ODI $\leq 15$ was $31 \%$. Further subgroup analysis showed no variation in score improvements age category ( $>$ vs. < age 65), number of implants used ( 2 vs. 3 ), sex, or prior lumbar fusion.

Using repeated measures analysis of variation, the mean improvement in VAS pain scores was slightly smaller in patients with SCS by an average of 0.4 points difference $(p=0.047)$ (Fig. 3) and slightly smaller in patients who had undergone staged bilateral procedures (average of 1.3 points difference, $p<0.0001)$. Similarly, improvement in mean ODI was slightly smaller in patients with SCS (2 points, $p=0.035)$ and those undergoing staged bilateral treatment (7.5 points, $p<0.0001)$.
CT scans, performed in 105 patients at 12 months, showed reabsorption along one or more implants in $16 \%$ of cases. However, no breaks and no pullouts of implants had occurred. Reabsorption rate was not affected by bilaterality of surgery. Generally, ossification around the implants could be detected at 1 year on CT scans, but a complete ossification of the target joint generally took 2 years or longer. No patient underwent revision as a result of recurrent pain or radiolucencies seen on CT scan. 
Fig. 3 Proportion of patients with VAS pain level $\leq 2$ or $\mathrm{ODI} \leq 15$ at 12 months by subgroup. Light gray $=$ no or unilateral, black $=$ yes or bilateral

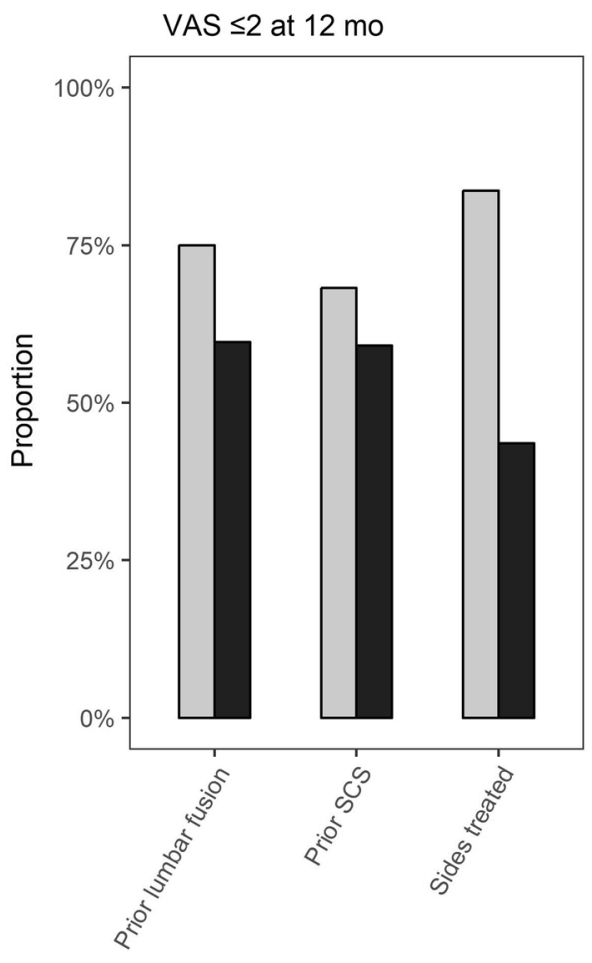

\section{Discussion}

We report here one of the largest consecutive cohorts of patients with refractory low back and leg pain diagnosed as SIJ pain and treated by minimally invasive SIJ fusion with triangular titanium implants. The SIJ joint has recently attracted much attention as a significant contributor to low back and leg pain, and interest in surgical treatment of the condition is growing. Using one of the first commercially available implants in Europe, we observed statistically significant, clinically important, and durable improvements in both SIJ pain (measured using a standard VAS) and disability (measured with ODI) after SIJ surgery. Moreover, the minimally invasive procedure for SIJ fusion with triangular implants was exceptionally safe in our hands and generally took less than 20 min to perform.

Careful diagnosis of the SIJ as the main source of low back pain is extremely important in the context of SIJ fusion, as only SIJ-generated pain will benefit from a surgical fusion. We have employed an established clinical algorithm that is well tested in previous retrospective and prospective studies. Also, functional diagnosis using fluoroscopy-controlled SIJ infiltrations to confirm the SIJ as a pain generator was employed preoperatively in all cases. All patients in our group had marked acute reduction in pain after SIJ infiltration with local anesthetic and/or corticosteroids, sometimes lasting only for a few hours. For all of our cases, prior nonsurgical therapies, such as physical therapy, steroid and/or local anesthetic injections and oral analgesics, had failed to provide sufficient long-term relief of pain and disability. We note that preoperative CT imaging is important to determine possible variations in SIJ shape and size that could affect implant trajectories, as well as the amount of degenerative changes and the bone density of the ilium and sacrum.

A large proportion of our patients had previous lumbar fusion surgery, a known risk factor for SIJ pain [19]. Degeneration of the SIJ after lumbar fusion is likely to result from increased force transmission across the SIJ due to permanent stabilization of the lumbar spine. SIJ pain after long fusions to the sacrum, which increase stress on the SIJ, also occurs commonly [20]. In our study, the relationship was high, with $64 \%$ of our cohort having a history of prior instrumented lumbar spine fusion. In the setting of known SIJ degeneration at the time of lumbar fusion, low back pain after lumbar fusion is highly likely to emanate from the SIJ.

The high prevalence of prior lumbar fusion in our cohort reflects to a large degree our center's focus on therapy-resistant chronic pain, and the associated frequency of lumbar fusions performed by ourselves. We were gratified to see that patients with SIJ pain associated with prior lumbar fusion derived substantial benefit from SIJ fusion. Indeed, we did not observe clinically important differences in SIJ pain or SIJ disability relief between those patients with and without a history of prior lumbar fusion. The lack of impact of prior lumbar fusion on pain and disability responses to SIJ fusion is consistent with other studies [21, 22]. Prior lumbar fusion done by us or others improved low back pain resulting from lumbar instability, osteochondrosis, disk herniation, 
neuroforaminal stenosis, lumbar spinal canal stenosis, or any combination of the above pathology. In clinical terms, the SIJ pain we diagnosed and treated was mostly subsequent to lumbar fusion. In addition, lumbar spinal pain is mostly of neuropathic origin, while SIJ pain is nociceptive in its nature. In rare cases, SIJ pain was present concomitantly at the time of initial neuropathic pain diagnosis and treatment, but was the minor component in our pain analysis, as proven by infiltrations of the lumbar facet joints and periradicular injections of affected lumbar nerve roots, which resulted in far greater pain relief than with SIJ infiltrations. It was therefore decided to first treat the major nociceptive pain component by lumbar fusion, and to address SIJ pain later, if clinically necessary.

Currently, functional diagnostics by infiltration of the SIJ joints is not a compulsory component of the diagnostic algorithm for lumbar spinal pathology. While diagnostic workup remains an individualized clinical decision, based on historical and physical examination findings, in our opinion, clinical and functional SIJ diagnostics should be added to the standard workup prior to instrumented lumbar fusion.

The body of knowledge on the relationship between SIJgenerated and lumbar spinal-generated pains and on their clinical and functional differentiations is still rather limited and to some extent arbitrary and needs to be greatly enlarged and standardized in order to deliver clear-cut answers on the relationship between lumbar versus SIJ pain.

Specific to our group was the high percentage of patients with an implanted SCS device (23\%). SCS was used primarily to address therapy-resistant chronic back and leg pain which was not responding to conservative treatments and which in some cases increased in intensity after one or more surgical procedures at the lumbar and/or lower thoracic spine. SCS appears to significantly reduce chronic neuropathic back and leg pain resulting from cauda equina and nerve root compression, fibrosis, or other mechanical irritation, but seems to provide little benefit for SIJ pain, which is commonly classified as joint pain and therefore as predominantly nociceptive pain. In our cohort, patients with implanted SCS devices had responses to SIJ fusion that were nearly the same as those without such devices. This suggests that SIJ fusion may serve as "rescue therapy" in some SCS patients, which otherwise would have counted as SCS failures because of the uncontrollable low back pain. Furthermore, we strongly suggest that screening for SIJ pain should be included in the workup of every candidate for SCS implantation.

A substantial portion of our patients underwent bilateral staged procedures. Bilateral cases improved significantly but, perhaps not surprisingly, had somewhat less improvement compared to those undergoing unilateral SIJ fusion. Bilateral cases were younger on average. In this subgroup, the second procedure followed the first procedure by, on average, 8 months. By month 12, mean improvements in
SIJ pain in the bilateral group were similar to those at 3 months (i.e., a similar postoperative time point) in the unilateral group. Nonetheless, responses in bilateral cases were clinically important and should not be discounted. Further follow-up of the bilateral SIJ fusion group may help to determine whether this group derives similar benefit compared with those undergoing unilateral SIJ fusion.

The mechanism of pain relief with the triangular titanium implants seems to be twofold: Early pain relief likely occurs as a result of immediate surgical joint stabilization and late pain relief as a result of both surgical stabilization and long-term fusion. Proper implant positioning is probably very important in achieving pain relief although this was not specifically investigated in our study. However, the fact that we routinely investigate implant positioning using postoperative $\mathrm{CT}$ scans, combined with the fact that there were no removals or redo surgeries, testifies to the high quality of the surgical procedure.

Potential explanations for inadequate pain relief after SIJ fusion include secondary loosening of the implants, inaccurate diagnosis, and the presence of concomitant disease (e.g., osteoarthritis, lumbar facet arthropathy) that can present in a manner similar to SIJ pain.

Although our study is retrospective, it provides high quality clinical data. All participants failed prior conservative treatments, and all were carefully and rigorously screened against predetermined eligibility criteria. All patients underwent structured follow-up visits in our clinic. The results of surgery represent the prospective experience of a single senior neurosurgeon with a large workload of SIJ fusions (typically 2-3 cases per week).

The 12-month follow-up rate in our group was high. The primary study limitations were lack of a concurrent control group treated non-surgically and the subjective nature of the used patient-reported outcomes. The significant regression of SIJ pain and disability observed in this study would be highly unusual with continued non-surgical care, as most patients had previously experienced no significant symptom improvement from non-surgical treatments provided. Two randomized controlled trials with identical enrollment criteria and using the same device and surgical technique showed very good pain relief in the surgical group and significantly less improvements in the control group without surgery $[13,14]$. Four-year followup from prospective trials conducted in the USA has also shown durable pain and disability relief [23].

Another potential limitation of the present study is lack of X-ray follow-up. However, 105 subjects underwent 1 -year CT scans, which showed bone growth around the implants across the joint and bone apposition to implants within the sacrum and ilium.

Taken together with the exceptionally low device- and procedure-related adverse events and side effects, the degree 
of SIJ pain relief observed in our study combined with the improvements in disability provides strong evidence that the benefits of the procedure outweigh the risks for most patients, at least in the 12-month follow-up.

\section{Conclusions}

In a standard clinical practice, pain from the SIJ can be identified by careful clinical and functional diagnostic procedure and can be effectively treated with minimally invasive implantation of triangular titanium implants. Prior lumbar fusion is frequent in patients with SIJ pain and does not negatively impact the effectiveness of SIJ fusion.

\section{Compliance with ethical standards}

Conflict of interest NGR has received speaker honorarium and consultancy fees from SI-Bone Germany GmbH.

Open Access This article is distributed under the terms of the Creative Commons Attribution 4.0 International License (http://creativeco mmons.org/licenses/by/4.0/), which permits unrestricted use, distribution, and reproduction in any medium, provided you give appropriate credit to the original author(s) and the source, provide a link to the Creative Commons license, and indicate if changes were made.

\section{References}

1. Bernard TN, Kirkaldy-Willis WH (1987) Recognizing specific characteristics of nonspecific low back pain. Clin Orthop Relat Res 217:266-280

2. Schwarzer AC, Aprill CN, Bogduk N (1995) The sacroiliac joint in chronic low back pain. Spine 20:31-37

3. Maigne JY, Aivaliklis A, Pfefer F (1996) Results of sacroiliac joint double block and value of sacroiliac pain provocation tests in 54 patients with low back pain. Spine 21:1889-1892

4. Irwin RW, Watson T, Minick RP, Ambrosius WT (2007) Age, Body Mass Index, and gender differences in sacroiliac joint pathology. Am J Phys Med Rehabil 86:37-44. https://doi. org/10.1097/PHM.0b013e31802b8554

5. Sembrano JN, Polly DW (2009) How often is low back pain not coming from the back? Spine 34:E27-E32. https://doi. org/10.1097/BRS.0b013e31818b8882

6. Cher D, Polly D, Berven S (2014) Sacroiliac joint pain: burden of disease. Med Devices (Auckl) 7:73-81. https://doi.org/10.2147/ MDER.S59437

7. Cher DJ, Reckling WC (2015) Quality of life in preoperative patients with sacroiliac joint dysfunction is at least as depressed as in other lumbar spinal conditions. Med Devices (Auckl) 8:395403. https://doi.org/10.2147/MDER.S92070

8. Petersen T, Laslett M, Juhl C (2017) Clinical classification in low back pain: best-evidence diagnostic rules based on systematic reviews. BMC Musculoskelet Disord 18:188. https:// doi.org/10.1186/s12891-017-1549-6

9. Szadek KM, van der Wurff P, van Tulder MW et al (2009) Diagnostic validity of criteria for sacroiliac joint pain: a systematic review. J Pain 10:354-368. https://doi.org/10.1016/j.jpain 2008.09.014

10. Painter CF (1908) Excision of the os innominatum. Arthrodesis of the sacro-iliac synchrondrosis. Boston Med Surg J 159:205-208

11. Smith-Petersen MN (1921) Arthrodesis of the sacroiliac joint. A new method of approach. J Bone Joint Surg Am 3:400-405

12. Buchowski JM, Kebaish KM, Sinkov V et al (2005) Functional and radiographic outcome of sacroiliac arthrodesis for the disorders of the sacroiliac joint. Spine J 5:520-528. https://doi. org/10.1016/j.spinee.2005.02.022; (discussion 529)

13. Polly DW, Swofford J, Whang PG, et al (2016) Two-year outcomes from a randomized controlled trial of minimally invasive sacroiliac joint fusion versus non-surgical management for sacroiliac joint dysfunction. Int J Spine Surg 10:Article 28. https:// doi.org/10.14444/3028

14. Dengler J, Kools D, Pflugmacher R et al (2017) 1-Year results of a randomized controlled trial of conservative management versus minimally invasive surgical treatment for sacroiliac joint pain. Pain Phys 20:537-550

15. Duhon BS, Bitan F, Lockstadt H et al (2016) Triangular titanium implants for minimally invasive sacroiliac joint fusion: 2-year follow-up from a prospective multicenter trial. Int J Spine Surg 10:Article 13. https://doi.org/10.14444/3013

16. Vanaclocha V, Herrera JM, Sáiz-Sapena N et al (2018) Minimally invasive sacroiliac joint fusion, radiofrequency denervation, and conservative management for sacroiliac joint pain: 6-year comparative case series. Neurosurgery 82:48-55. https://doi.org/10.1093/ neuros/nyx 185

17. Graham Smith A, Capobianco R, Cher D et al (2013) Open versus minimally invasive sacroiliac joint fusion: a multi-center comparison of perioperative measures and clinical outcomes. Ann Surg Innov Res 7:14. https://doi.org/10.1186/1750-1164-7-14

18. Fairbank JC, Pynsent PB (2000) The Oswestry Disability Index. Spine 25:2940-2952; (discussion 2952)

19. DePalma MJ, Ketchum JM, Saullo TR (2011) Etiology of chronic low back pain in patients having undergone lumbar fusion. Pain Med 12:732-739. https://doi.org/10.111 1/j.1526-4637.2011.01098.x

20. Diesing D, Franke J, Tschoeke SK et al (2018) Persistent iliosacral joint syndrome following instrumentation to the sacropelvis in patients with adult spinal deformity. J Neurol Surg A Cent Eur Neurosurg. https://doi.org/10.1055/s-0038-1655732

21. Rudolf L (2013) MIS fusion of the SI joint: Does prior lumbar spinal fusion affect patient outcomes? Open Orthop J 7:163-168. https://doi.org/10.2174/1874325001307010163

22. Dengler J, Duhon B, Whang P et al (2017) Predictors of outcome in conservative and minimally invasive surgical management of pain originating from the sacroiliac joint: a pooled analysis. Spine 42:1664-1673. https://doi.org/10.1097/BRS.0000000000002169

23. Darr E, Cher D (2018) 4-Year outcomes after minimally invasive transiliac sacroiliac joint fusion with triangular titanium implants. Med Devices (Auckl) 11:287-289. https://doi.org/10.2147/ MDER.S179003 


\section{Affiliations}

\section{Nikolai G. Rainov ${ }^{1} \cdot$ Reinhard Schneiderhan $^{1} \cdot$ Volkmar Heidecke $^{2}$}

1 MVZ Wirbelsäulenzentrum Taufkirchen b. München, Eschenstr. 2, 82024 Taufkirchen, Munich, Germany
2 Klinik für Neurochirurgie, Klinikum Augsburg, 86156 Augsburg, Germany 